\title{
Avances en el diagnóstico por ultrasonido endoscópico (USE)
}

\author{
Rodrigo Soto-Solis ${ }^{*}$
}

Centro Médico Nacional "20 de Noviembre"ISSSTE, Ciudad de México, México

\begin{abstract}
Resumen
Desde 1995 y por idea del Dr. Arturo Ballesteros, se inició el primer congreso llamado ECOS de la AGA que posteriormente daría lugar a ECOS Internacionales. Esta idea la han tomado varias asociaciones médicas, incluida la Asociación Mexicana de Endoscopia Gastrointestinal (AMEG), con la finalidad de acercar los principales y más trascendentes trabajos de los principales congresos internacionales a médicos latinoamericanos. Esta es una revisión analítica de los trabajos libres presentados en la United European Gastroenterology Week 2018 (UEG 2018) y en la Digestive Disease Week 2019 (DDW 2019), en donde el Ultrasonido Endoscópico (USE) se usó como modalidad diagnóstica primaria. Los trabajos evaluados se dividieron para su mejor comprensión en aquellos con utilidad para el escrutinio de cáncer de páncreas, aquellos en los que se utilizó la elastografía por USE, el medio de contraste, la endomicroscopia confocal láser y aquellos que evaluaron los nuevos métodos de adquisición de tejido.
\end{abstract}

Palabras clave: Ultrasonido endoscópico. Ultrasonido endoscópico con contraste. Elastografía por ultrasonido endoscópico. Agujas histológicas para ultrasonido endoscópico. Endomicroscopía confocal láser.

\section{Introducción}

El Ultrasonido Endoscópico (USE) es una herramienta útil para el diagnóstico, el estadiaje de la enfermedad maligna y el tratamiento de las enfermedades hepatopancreatobiliares. En los últimos años ha habido avances indudables en su potencial para el diagnóstico, principalmente en la adquisición de tejido con nuevas agujas, así como tecnologías complementarias como la elastografía, el uso de medio de contraste, la endomicroscopia confocal láser a través de aguja y la adquisición de tejido.

\section{Objetivo}

Realizar una revisión analítica y crítica de los trabajos de mayor trascendencia presentados en la United
European Gastroenterology Week 2018 (UEG 2018) y en la Digestive Disease Week 2019 (DDW 2019), en donde se haya utilizado al Ultrasonido Endoscópico (USE) como modalidad diagnóstica primaria, desde un punto de vista novedoso.

Número de trabajos incluidos: 12. Evaluados: DDW = 28 carteles y 7 presentaciones orales; UEG $=29$ carteles y 3 presentaciones orales.

\section{Escrutinio de cáncer de páncreas}

El primer estudio es un programa multicéntrico italiano de escrutinio para cáncer de páncreas en pacientes de alto riesgo ${ }^{1}$. Este estudio incluyó: individuos con 3 o más familiares con cáncer de páncreas (primer, segundo o tercer grado); individuos con al menos 2 familiares con cáncer de páncreas de los

\section{Correspondencia:}


cuáles uno es de primer grado, portadores de mutación BRCA o p16 con al menos un familiar de primer o segundo grado diagnosticado con adenocarcinoma de páncreas e individuos con pancreatitis hereditaria o Síndrome de Peutz-Jegher. El programa consistió en la realización de al menos colangiorresonancia (MRCP) anual; se indicó USE a aquellos individuos que no quisieran o no pudieran realizarse dicho estudio. Se realizó un análisis univariado y multivariado para identificar factores de riesgo para la detección de lesiones premalignas o malignas. Se evaluaron 189 pacientes, 174 con MRCP y 15 con USE. Se realizó USE en 9 pacientes por MRCP sospechosa. De estos, 123 casos eran de tipo familiar, 61 sindromáticos y 5 pancreatitis hereditaria. Se encontraron 55 anormalidades, siendo detectadas 44 por MRCP. Se detectaron 6 adenocarcinomas del páncreas por USE, de los cuales 2 fueron sometidos a cirugía con intención curativa, 2 eran localmente avanzados y 2 metastásicos. Se detectaron 4 anormalidades más por USE y este fue normal en 5 pacientes. Dos pacientes fueron operados (Peutz Jegher y pancreatits hereditaria), en uno se documentó un carcinoma invasor y en el otro displasia de bajo grado en un IPMN mixto. La tasa de malignidad fue mayor en el grupo no familiar. En el análisis multivariado fumar, tener más de dos familiares con adenocarcinoma pancreático y tener más de 50 años resultaron factores independientes para presentar una lesión premaligna o maligna. Los autores concluyen que, en el reporte inicial, se encontraron con una alta incidencia de malignidad pancreática $(3.7 \%)$ y la mayoría de estos casos se encuentran en una etapa avanzada. La cohorte con Peutz-Jegher mostró un mayor riesgo (10\%). El segundo estudio es israelí y en él evaluaron la factibilidad del escrutinio con ultrasonido endoscópico de los pacientes de alto riesgo para cáncer de páncreas ${ }^{2}$. Se incluyeron 123 pacientes evaluados entre 2008 y 2018 de forma anual o bienal. Se hizo un análisis retrospectivo de estos casos, encontrando una edad media de 57.2 años, el $40 \%$ de los USE fueron normales y 2 pacientes fueron sometidos a cirugía pancreática. Los autores concluyen que se requiere más evidencia para establecer programas de escrutinio útiles.

\section{Elastografía por use}

La elastografía es una herramienta no invasiva que evalúa la dureza o la elasticidad tisular en tiempo real; esta tecnología ha mostrado grandes avances en el estudio de las masas pancreáticas, pues su nivel de dureza ha sido evaluado tanto de forma cualitativa como cuantitativa. El grupo del Dr. Robles-Medrana de Ecuador evaluó la elastografía hepática y esplénica para predecir hipertensión portal y cirrosis ${ }^{3}$. Se trata de un estudio de casos y controles evaluados de forma prospectiva. En el grupo de casos se incluyeron pacientes con cirrosis y en el grupo control sujetos sin cirrosis con alguna lesión submucosa que requiriera de evaluación endosonográfica. Se utilizó un ecoendoscopio Pentax EG 3870UTK con una consola Hitachi AVIUS. Se evaluaron la dureza hepática y esplénica en 10 ocasiones y se complementó con el diámetro de la vena ácigos, la velocidad media y el volumen de esta. Los autores incluyeron 61 pacientes de los cuales 32 presentaban cirrosis. Se compararon los resultados de la elastografía transitoria transcutánea (TE) y la elastografía por USE. Se encontró una asociación directa entre el radio de tensión rigidez hepática (LSMSR) y la elastografía transitoria. Se obtuvo un área bajo la curva igual o mayor al $80 \%$ en LSM-SR con valor de 5.2, en el radio del histograma de la medición de la rigidez hepática (LSM-SH) con valor de 88.8, así como para las mediciones de la rigidez esplénica (SSM), SSM-SR de 7.5 y SSM-SH de 39.9. Para predecir cirrosis e hipertensión portal, la sensibilidad, especificidad, valor predicitivo positivo y valor predictivo negativo fueron los siguientes: LSM-SR 84.3, 82.8, 84.4 y $82.8 \%$; LSM-SH $87.5,69,75.7$ y $83.3 \%$; SSM-SR $87.5,69,75.7$ y $83.3 \%$; SSM-SH $56.3,89.7$, 85.7 y $65 \%$. Por ello, concluyen que la elastografía por USE puede ser útil para predecir cirrosis e hipertensión portal.

\section{Ultrasonido endoscópico con contraste armónico (USE-C)}

En los últimos congresos internacionales se presentaron varios trabajos que abordan esta tecnología. En el metaanálisis japonés de Yamashita, et al. ${ }^{4}$ incluyeron 9 estudios para un total de 887 pacientes. Se evaluó la capacidad del USE-C para distinguir entre cáncer de páncreas y lesiones no neoplásicas. La sensibilidad acumulada fue del $93 \%$ (95\% IC, 0.91-0.95) y la especificidad del $80 \%$ (95\% IC, 0.75-0.85). Por ello concluyen que el análisis cualitativo del patrón de reforzamiento es útil en el diagnóstico de cáncer de páncreas. De forma interesante y complementaria, el grupo coreano de Jun-Ho Choi evaluó la utilidad del USE-C en la ablación de lesiones por radiofrecuencia (ARF) 5 . Se trata de un estudio prospectivo donde evaluaron 19 
pacientes con tumores sólidos abdominales en quienes se realizó ARF por USE. La mediana de sesiones de ARF fue de 2. Al evaluar post-ARF las lesiones, 7 lesiones no mostraron realce intratumoral y 12 tumor residual. Estas 12 lesiones se trataron con USE-C en tiempo real en sesiones repetidas hasta lograr respuesta completa (esto se logró en 15 casos). En el seguimiento (36 meses) se documentaron 2 recurrencias. Los autores concluyeron que USE-C puede ser útil para evaluar respuesta y guiar tratamiento con ARF.

\section{Endomicroscopia confocal láser (CLE)}

En la UEG week 2018 se presentó en formato de póster un modelo exvivo para la evaluación con endomicroscopia confocal láser del cáncer de páncreas ${ }^{6}$. En este estudio se evaluaron 12 casos posterior a la resección quirúrgica del adenocarcinoma pancreático. Se utilizó acriflavina como contraste y se evaluaron mediante endomicroscopia confocal láser (CLE) con varias minisondas. Posterior a esto se evaluaron histopatológicamente y por inmunohistoquímica. Se encontró utilidad en el modelo exvivo para utilizar CLE para diferenciar entre tejido tumoral y no tumoral en adenocarcinoma de páncreas. Más allá de esto, en la DDW 2019 se presentaron los resultados de la evaluación invivo de neoplasias quísticas del páncreas a través de una punción guiada por USE ${ }^{7}$. En este estudio se incluyeron 26 estudios para establecer en una forma ordenada los parámetros de mayor utilidad al evaluar estos quistes pancreáticos.

Los dos parámetros de mayor utilidad fueron el grosor y lo oscuro del epitelio; esto se llevó un paso más allá, al utilizar un programa de computadora para ayudar a detectar estos parámetros. La sensibilidad y especificidad para la predicción de displasia de alto grado o neoplasia avanzada en neoplasia papilar intraductal fue de 88 y $100 \%$ para el observador humano y de 70 y $90 \%$ para el diagnóstico ayudado por computadora, respectivamente. Por otro lado, el grupo de Mount Sinai en Nueva York presentó los resultados de la evaluación por CLE y la toma de biopsia con micropinza de biopsia a través de una aguja $19 \mathrm{~g}$ de $\mathrm{USE}^{8}$. Se trata de un estudio retrospectivo donde evaluaron la utilidad diagnóstica y su impacto en la conducta terapéutica. Se evaluaron 44 quistes en 44 pacientes, la edad media fue de 66 años y el tamaño promedio fue de $33.5 \mathrm{~mm}$. El éxito técnico fue del $100 \%$ para la biopsia guiada por USE; sin embargo, se obtuvo un diagnóstico citológico en $15.9 \%$ con líquido suficiente para su análisis en el $59.1 \%$. La biopsia con micropinza requirió una media de 3 pases (1-5) con obtención de muestra suficiente en $88.6 \%$. CLE fue exitosa en el $97.7 \%$. Hubo un evento adverso grave derivado de la infección de un quiste. La eficacia diagnóstica mejoró con una diferencia estadísticamente significativa para la micropinza $(75 \%)$ y para la endomicroscopia confocal láser (84.1\%). No hubo diferencia entre ambas tecnologías o en su uso combinado. Además de esto, demostraron un cambio en la conducta clínica cuando lo compararon con el estándar de referencia.

\section{Adquisición de tejido}

En el estudio del grupo de Vinay Dhir ${ }^{9}$ presentado como cartel en la DDW 2019, se comparó la biopsia de lesiones sólidas con la aguja Acquire ${ }^{\circledR}$ contra la citología tradicional. Se trata de un estudio retrospectivo donde incluyeron 207 pacientes (83 con aguja histológica y 124 con citología estándar). En el grupo con aguja histológico se hubo necesidad de repetir 3 biopsias y en el de citología 17. Los autores concluyeron que las nuevas agujas facilitan el diagnóstico de lesiones benignas y malignas y deben reemplazar a las agujas tradicionales. Hubo múltiples estudios evaluando las diferencias entre las nuevas agujas histológicas $^{10}$; sin embargo, de mayor interés me parecieron 2 métodos de adquisición celular más recientes, por un lado el uso de la micropinza"11 a través de una aguja $19 \mathrm{~g}$ y por otro, la búsqueda de células tumorales en la circulación portal (PV-CTCs) ${ }^{12}$. La micropinza demostró utilidad y reproducibilidad en 30 casos. Asimismo, ayudó al diagnóstico de $64 \%$ de lesiones que con otros métodos fueron indeterminadas. En cuanto a la obtención de células tumorales de la circulación portal, se incluyeron 17 pacientes en quienes se obtuvo sangre portal por punción guiada por USE. Al evaluarlas como factor pronóstico en cáncer hepatopancreatobiliar, los autores encontraron una correlación entre un número alto de PV-CTCs y la progresión tumoral. Concluyen que la búsqueda de estas células puede traer avances en la estadificación y tal vez en el tratamiento de estos pacientes.

\section{Conclusiones}

El último año ha sido productivo en torno al ultrasonido endoscópico. Considero de particular interés los nuevos métodos para el diagnóstico de las lesiones quísticas, particularmente el uso de la micropinza y la endomicroscopia confocal láser a través de una aguja 
de punción. Sin duda estos avances verán cosechados sus frutos en el diagnóstico certero de los pacientes con lesiones premalignas o malignas, principalmente el adenocarcinoma del páncreas.

\section{Conflicto de intereses}

\section{No existen conflictos de interés. Sin patrocinio de la} industria.

\section{Bibliografía}

1. Paiella S, Capurso G, Cavestro GM, Butturini G, Pezzilli R, Salvia R, Signoretti M, Crippa S, Carrara S, Frigerio I, Bassi C, Falconi M, lannicelli E, Giardino A, Zuppardo R, Laghi A, Laghi L, Frulloni L, Zerbi A. First-round screening results in subjects at risk of pancreatic cancer from the AISP (Italian Association for the Study of the Pancreas) Registry. Presentación oral (OP015), UEG 2018, Viena, Au.

2. Lachter J, Half E, Khamaysi I, Yassin KA. Screening to detect precursor lesions of pancreatic adenocarcinoma in high-risk individuals experienced from Rambam Health Care Campus. Presentación en poster (P1366), UEG 2018, Viena, Au.

3. Robles-Medranda C, ValeroM, Puga-Tejada M, Oleas R, Autran-Nebel J, De Britto S, Ospina-Arboleda J, Soria-Alcivar M, Alvarado-Escobar H, Del Valle R, Pitanga Lukashok H. P0852 utility of liver and spleen eus-elastography as a diagnostic tool for predicting liver cirrhosis and portal hypertension: a prospective case-control study. Presentación en póster (P0852), UEG 2018, Viena, Au.
4. Yamashita Y, Shimokawa T, Napoleon B, Fusaroli P, Gincul R, Kudo M, Kitano M. Value of contrast-enhanced harmonic eus with enhancement pattern for diagnosis of páncreatic cancer: a meta-analysis. Presentación en póster (Tu 1344), DDW 2019, San Diego, EUA.

5. Choi JH, Seo DW, Song TJ, Park DH, Lee SS, Lee SK, Kim MH. Utility of contrast enhanced harmonic eus in radiofrequency ablation for solid abdominal tumors. Presentación en póster (384), DDW 2019, San Diego, EUA.

6. Ungureanu B, Pirici D, Dima SO, Popescu I, Gheona DI, Ciurea T, Surlin V, Saftoiu A. Probe based confocal laser endomicroscopy assessment of pancreatic ductal adenocarcinoma, $\mathrm{n}$ ex-vivo study with pathological correlation. Presentación en poster (P0735), UEG 2018, Viena, Au.

7. Krishna S. Qualitative derivation and quantitative prediction of dysplasia in intraductal papillary mucinous neoplasms using eus-guided needle based confocal laser endomicroscopy. Presentación oral (4280), Advances in EUS and ERCP. DDW 2019, San Diego, EUA.

8. Cheesman A. EUS-Guided microforceps biopsy and needle-based confocal laser endomicroscopy significantly improve the diagnostic yield and have major impact on clinical management of pancreatic cystic lesions. Presentación oral (5525), Maximazing the Clinical Utility of EUS Guided Sampling. DDW 2019, San Diego, EUA.

9. Shah R, Banka NH, Dhir V. Endoscopic ultrasound guided biopsy using franseen needle: coparison with EUS Guided fine needle aspiration. Presentación en póster (Tu1374), DDW 2019, San Diego, EUA.

10. Cho E, Park CH, Kim TH, Cho CM, Seo DW, Choi JH, Moon SH, Kim J. Prospective, randomized, multicenter clinical trial comparing 25-gauge and 20-gauge procore needles for endoscopic ultrasound-guided sampling of solid pancreatic lesions. Presentación en póster (P0116), UEG 2018, Viena, Au.

11. Wilen J, Visrodia K, Lan G, Seth A, Poneros JM, Gonda TA. The feasibility and value of cyst wall biopsy using micro forceps in the diagnosis of pancreatic cysts. Presentación en póster (Tu1389), DDW 2019, San Diego, EUA.

12. Swel E. High numbers of portal venous circulating tumor cells acquired via EUS provide prognostic assistance for progression free survival in pancreaticobiliary cancers. Presentación oral (3560), EUS-Based Innovation in the Diagnosis and Treatment of Pancreatic Cancer. DDW 2019 , San Diego, EUA. 